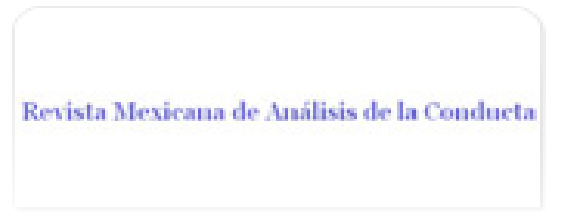

Revista Mexicana de Análisis de la Conducta ISSN: 0185-4534

editora@rmac-mx.org

Sociedad Mexicana de Análisis de la Conducta México

Flores Montañez, Nidia; Santoyo Velasco, Carlos Estabilidad y cambio de las relaciones sociales entre niños: Análisis de mecanismos funcionales Revista Mexicana de Análisis de la Conducta, vol. 35, núm. 1, junio, 2009, pp. 59-74

Sociedad Mexicana de Análisis de la Conducta Guadalajara, México

Disponible en: http://www.redalyc.org/articulo.oa?id=59311416004

- Cómo citar el artículo

- Número completo

- Más información del artículo

- Página de la revista en redalyc.org

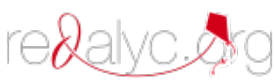

Sistema de Información Científica Red de Revistas Científicas de América Latina, el Caribe, España y Portugal Proyecto académico sin fines de lucro, desarrollado bajo la iniciativa de acceso abierto 


\title{
ESTABILIDAD Y CAMBIO DE LAS RELACIONES SOCIALES ENTRE NIÑOS: ANÁLISIS DE MECANISMOS FUNCIONALES
}

\author{
STABILITY AND CHANGE OF SOCIAL RELATIONS BETWEEN \\ CHILDREN: ANALYSIS OF FUNCTIONAL MECHANISM
}

\author{
NIDIA FLORES MONTAÑEZ Y CARLOS SANTOYO VELASCO ${ }^{1}$ \\ UNIVERSIDAD NACIONAL AUTÓNOMA DE MÉXICO
}

\begin{abstract}
RESUMEN
En este estudio, se examinó la estabilidad de las relaciones sociales y su asociación con las propiedades y mecanismos implicados en éstas. Participaron 21 niños de un grupo natural del quinto grado de primaria, con un seguimiento de tres periodos a lo largo de un año. A partir del empleo de procedimientos de nominación mutua de amistad, mapas socio-cognoscitivos compuestos y la observación de interacciones sociales, según su estabilidad se formaron tres grupos de relaciones: estables, de mediana vida y breves. Se identificaron y contrastaron las propiedades de inicio y tiempo de interacción, así como los mecanismos de efectividad, correspondencia y reciprocidad social de estos grupos. Los resultados muestran que existe una asociación entre la estabilidad de las relaciones sociales y las propiedades de inicio y tiempo de interacción, así como con el mecanismo de reciprocidad social. Así mismo, la convergencia entre los tres procedimientos resultó una estrategia valiosa para identificar el desarrollo de las relaciones sociales en escenarios naturales.
\end{abstract}

Palabras clave: Relaciones sociales, estabilidad, reciprocidad, redes sociales, niños de primaria.

1. C. S. V. agradece el apoyo del CONACyT: 057327 y de PAPIIT:3000409. Esta investigación es parte de la tesis doctoral de la primera autora. Agradecemos los comentarios de L. Colmenares, B. Mendoza y de los revisores anónimos. Correspondencia: carsan@servidor.unam.mx; montaneznf@yahoo.com.mx.

Recibido: 13 de marzo. Revisado: 27 de febrero. Aceptado: 20 de abril de 2009 


\begin{abstract}
The aim of this research was to evaluate the association between the properties and the stability of social relations and their related mechanisms. Twenty one children from a fifth grade group of elementary school were interviewed and observed three times over a year. Three groups were constituted derived from friendship mutual nomination, social cognitive maps and the observation of social interactions, where the stability of their relations was the criteria. The dyadic categories of analysis were stable, average and short relations. Properties of initiating acts, peers time allocation, social effectiveness, social responsiveness and reciprocity were evaluated. Data show an association between the stability of social relations and initiating acts, dyadic time allocation and reciprocity. The convergence of the three main procedures of data collection proved a valuable strategy to identify the development of social relations in natural settings.

Key words: Social relationships, stability, reciprocity, social networks, elementary school children.
\end{abstract}

Para el análisis y seguimiento de las experiencias sociales entre niños, se distinguen tres niveles de complejidad social: las interacciones, las relaciones y los grupos sociales. Las interacciones se refieren a conductas de dos individuos, en donde la conducta de cada miembro es tanto respuesta como estímulo para la conducta del otro, como un proceso que implica control mutuo. Las relaciones como fenómeno diádico conllevan una historia de interacciones sociales, donde su curso está influido por interacciones previas y por expectativas de interacciones futuras. Los grupos implican estructuras que emergen de las características y patrones de las relaciones y de las interacciones, además de manifestar jerarquías y normas que definen y dan cohesión al grupo (Rubin, Bukowsky \& Parker, 1998).

Este trabajo se dirige al estudio de la estabilidad y cambio de las relaciones sociales, con dos metas sustantivas: describir por cuánto tiempo se mantienen las relaciones y evaluar los factores responsables.

Los procedimientos más empleados para valorar dicha estabilidad han sido la nominación unilateral y la nominación mutua de amistad. Sin embargo, estos procedimientos implican dos limitaciones. Con la nominación unilateral se corre el riesgo de medir sólo la preferencia de un niño y no una relación de amistad, por lo que algunos estudios optan por emplear nominaciones mutuas (Asher, Parker \& Walker, 1996). La segunda limitación es que las relaciones sociales se han entendido como sinónimo de amistad.

Las relaciones sociales refieren a una diversidad de vínculos y la amistad es sólo un tipo de relación, por lo que no deben ser consideradas como 
sinónimos. Sin embargo, el procedimiento de nominación mutua puede ser empleado en conjunto con otros procedimientos para identificar relaciones. Asumiendo que los grupos se componen de un conjunto de relaciones sociales, es posible emplear procedimientos sociométricos para identificar relaciones sin recurrir al concepto de amistad. Tales procedimientos son los mapas socio-cognitivos compuestos y los mapas socioconductuales.

En los mapas socio-cognitivos se pide a los niños que mencionen qué compañeros de su grupo "se juntan frecuentemente". La información proporcionada por cada niño se integra de manera que se identifican subgrupos de niños a partir del "consenso" (Gest, Farmer, Cairns \& Xie, 2003). Por su parte, los mapas socioconductuales se diseñan a partir de la conducta social de los niños en escenarios naturales, la cual se registra mediante metodología observacional para identificar agrupaciones de niños con base en el tiempo que dedican a interactuar. Al mismo tiempo, a través de técnicas de microanálisis existe la posibilidad de estudiar los mecanismos implicados en la regulación de las interacciones (Santoyo, 2006).

Se ha explorado si las características de los niños, como timidez/introversión, popularidad, agresión, ser víctima, la edad y el género se encuentran asociadas a la estabilidad de las relaciones sociales, sin encontrar correlaciones significativas entre estas variables y la estabilidad (Berndt \& Hoyle, 1985; Bukowski \& Newcomb, 1984; Wojslawowicz, Rubin, Burgess, Booth-LaForce $\&$ Rose-Krasnor, 2006). Pero se ha encontrado que en las escuelas que mantienen la configuración grupal año con año, se observa mayor estabilidad en las relaciones en comparación con las escuelas en donde los niños, con cada ciclo escolar, son reasignados a nuevos grupos (Berndt \& Hoyle, 1985).

En este trabajo, para evaluar aspectos conductuales vinculados con la estabilidad de las relaciones sociales, se asume que las interacciones sociales juegan un papel primordial en la formación, mantenimiento y disolución de las relaciones (Hinde, 1995).

Santoyo (2006) propone tres mecanismos funcionales que regulan las interacciones sociales: la efectividad, la correspondencia y la reciprocidad social. Para evaluar tales mecanismos, se requiere identificar quién inicia la interacción y analizar, secuencialmente, su resultado. Así, cuando el niño A inicia una acción hacia el niño $B$ y éste responde, se considera que $A$ muestra efectividad social, como una habilidad que produce una reacción en su ambiente social. Por otra parte, sí A responde a los actos de otros, se dice que exhibe correspondencia como una habilidad de responder a las demandas de su ambiente social y una forma de proporcionar reforzamiento a las iniciativas de otros. La reciprocidad se define como "una igualación en la proporción" o simetría de conductas (Santoyo, 2006), aunque para Gottman (1979) la reciprocidad implica interacciones diádicas en las cuales la persona A y la $B$ se refuerzan uno a otro en una proporción equitativa. 
Los estudios sobre el desarrollo social de niños con necesidades especiales, son un ejemplo de cómo operan la efectividad y la correspondencia social; pues se promueven y modelan conductas de inicio (invitaciones a jugar, a conversar) y de respuesta a las emisiones de otros, para incrementar el número de interacciones sociales en que estos niños se involucran (Kohler, Phillips, Hoyson, Davis, Donina, \& Rapp, 1995). El papel de la reciprocidad se ha observado también en el mantenimiento de patrones de agresión en niños preescolares y niños de primaria. Los hallazgos muestran que los niños con altas tasas agresión, suelen involucrarse en interacciones, donde reciben tasas similares de agresión a las que ellos provocan (Santoyo, Colmenares \& Figueroa, 2007).

El objetivo de este trabajo es estudiar la estabilidad de las relaciones sociales a partir del análisis de sus propiedades conductuales y de los mecanismos funcionales de efectividad, correspondencia y reciprocidad social.

\section{MÉTODO}

\section{Participantes}

Un grupo de 21 niños de quinto grado de primaria, conformado por doce niñas y nueve niños, con una edad promedio de 10 años al inicio del estudio. Dieciocho de los integrantes ya se conocían desde cuarto grado y tres niños se integraron en el quinto grado. Para la última fase del estudio sólo permanecieron 19 niños.

\section{Escenario}

Escuela primaria particular al oriente del Distrito Federal. Se contó con la autorización expresa de las autoridades correspondientes, bajo el acuerdo de guardar la confidencialidad de los participantes en el estudio. La escuela contaba con un grupo por grado, de manera que mantuvo la configuración grupal año con año.

\section{Instrumentos}

Entrevista semi-estructurada diseñada para la elaboración de mapas sociocognitivos e incluía las preguntas “¿Quiénes de tu salón se juntan mucho?” y “¿Quiénes son tus amigos?". Si en la nominación, el niño entrevistado, no se auto nombraba se preguntaba "¿Y tú con quien te juntas en la escuela?". Si faltaba alguien de nominar se preguntaba " $¿ Y$-nombre del compañero- con quién se junta?" (ver Cairns, Leung, Buchanan, \& Cairns, 1995).

Se utilizó el Sistema de Observación Conductual de las Interacciones Sociales (Espinosa, Blanco \& Santoyo, 2006), que es un sistema de categorías conductuales excluyentes y exhaustivas mediante el registro por intervalos 
de cinco segundos. Permite identificar actividades individuales, sociales y los niños involucrados. En esta investigación sólo se utilizaron las categorías relativas a la interacción social. Para este registro conductual se emplearon hojas de registro, lápices y cronómetros.

\section{Procedimiento}

La entrevista y el muestreo conductual se llevaron a cabo en tres cortes, uno al comenzar el quinto grado, el segundo a la mitad del ciclo escolar y el tercero al iniciar el sexto grado. El tiempo transcurrido entre cada corte fue de aproximadamente seis meses. Los niños fueron entrevistados individualmente en un periodo aproximado de diez minutos, en cada uno de los cortes.

Cada niño fue observado en la zona de juego durante el recreo por una pareja de observadores que desconocía los objetivos del estudio. Los observadores fueron capacitados en el sistema de observación hasta que alcanzaran una concordancia mínima de .80 en cuatro registros consecutivos. El índice Kappa promedio para la concordancia entre observadores fue de .81 durante los tres cortes. Además, se consideró el acuerdo entre observadores respecto a los agentes sociales identificados, con una concordancia del $90 \%$. Se obtuvieron para cada niño seis sesiones de 10 minutos en el corte uno y tres; en el corte dos se condujeron cinco sesiones de 10 minutos por niño.

\section{Criterios para la identificación de relaciones sociales}

A partir de las respuestas a la pregunta "¿quiénes son tus amigos?" se obtuvieron las relaciones sociales identificadas por nominación mutua. Si el niño A mencionaba como amigo al B, se confirmaba sí B mencionaba como amigo a $\mathrm{A}$. No se limitó el número de amigos ni el género a mencionar.

Con la información obtenida de la pregunta "¿Quiénes de tu salón se juntan mucho?", se construyeron los mapas socio-cognitivos compuestos con el siguiente procedimiento. En primer lugar, se construyó una matriz de recuerdo, colocando en las columnas a los niños entrevistados y en las filas a los niños nombrados, preservando el orden en que cada niño y grupo fue recordado. Posteriormente, con base en la matriz de recuerdo se generó una matriz simétrica de "co-ocurrencia", la cual contiene el número de veces que dos niños fueron nombrados como miembros de un mismo grupo (coocurrieron). La diagonal de esta matriz resume el número de veces que un niño fue nombrado como parte de un grupo.

Finalmente, se obtuvo una matriz de correlación a partir de la inter-correlación de las columnas de la matriz co-ocurrente. Se consideró que dos niños pertenecían a un mismo grupo si su correlación era $r \geq .50$ y significativa al .01. Con este criterio se agrupó a los niños y se representó la red social en el mapa socio-cognitivo compuesto (para detalles del procedimiento, ver Gest et al, 2003). En este trabajo, no se consideró al grupo como unidad, sino a 
las díadas de niños que lo conformaban. Así, de un grupo formado por cuatro niños que cubría el criterio señalado, se identificaban seis díadas de niños "que se juntan mucho", según el consenso del grupo, en donde un mismo niño podía pertenecer a más de una díada.

En cuanto al método de observación, de cada registro obtenido se identificaron los episodios y los agentes sociales implicados. Posteriormente se obtuvo la frecuencia de actos de inicio (emisiones, recepciones) y la frecuencia de intervalos (tiempo de interacción social) producto de tales inicios. Las relaciones se identificaron a partir del tiempo de interacción de cada díada, comparado con la duración promedio de las interacciones en el grupo. Sólo se consideró como relaciones sociales a aquellas donde la díada fue observada interactuando un $15 \%$ más que el promedio del grupo, con el objetivo de eliminar interacciones ocasionales (Smith \& Connolly, 1980).

Clasificación de relaciones según su estabilidad

A partir de las díadas identificadas por cada método, se formaron tres grupos de relaciones sociales según su estabilidad.

Grupo de relaciones "estables".- Compuesto de díadas identificadas en los tres cortes por un mismo método (nominación, observación o consenso). Así, una díada fue estable en nominación de amistad, sí fue identificada en los tres cortes por este método; la percepción que el grupo tenía acerca de una díada ("se juntan mucho") fue estable sí la díada fue identificada en los tres cortes por consenso grupal y, finalmente, una díada resultó estable sí fue observada en interacción social en los tres cortes.

Grupo de relaciones de "mediana vida".- Formado a partir de las relaciones identificadas en dos cortes, incluye díadas identificadas en los cortes uno y dos, dos y tres ó uno y tres.

Grupo de relaciones "breves".- Refiere a díadas que sólo fueron identificadas sólo en uno de los tres cortes.

\section{Obtención de índices funcionales}

Los índices funcionales de efectividad, correspondencia y reciprocidad se obtuvieron a partir de los actos de inicio y los intervalos de interacción de cada una de las díadas. La efectividad social se obtuvo dividiendo la frecuencia de actos de inicio -del niño focal- que fueron respondidos por el compañero (concluyeron en interacción) entre la frecuencia de actos de inicio totales (concluyeran o no en interacción). La correspondencia social se calculó a partir de las respuestas del niño focal a los actos de inicio de su compañero entre el total de actos de inicio de su compañero. Estos índices producen valores entre cero y uno, donde valores cercanos a uno sugieren máxima efectividad o correspondencia. Al azar se eligió a uno de los miembros de la diada como niño focal (niño a). 
La reciprocidad se obtuvo comparando la frecuencia relativa de inicios con la frecuencia relativa de intervalos. Para obtener la frecuencia relativa de inicios se dividió el total de actos de inicio del niño focal (la) dirigidos al par entre el total de inicios generado por ambos miembros de la díada $(\mathrm{l} a+\mathrm{lb})$, y para obtener el tiempo relativo se dividió el tiempo de interacción que el niño focal generó con sus inicios (Ta) entre el tiempo de interacción que ambos compañeros generaron $(\mathrm{Ta}+\mathrm{Tb})$.

\section{RESULTADOS}

Con el método de nominación se identificaron seis relaciones "estables", diez de "mediana vida" y 22 relaciones "breves". Por el método de consenso se identificaron seis, diez y 20 , respectivamente; mientras que con el método de observación se identificaron nueve "estables", nueve de "mediana vida" y 31 relaciones "breves".

En general, los datos mostraron probabilidades bajas de que una relación sea "estable", con valores de .16, .17 y .18 en los métodos de nominación, consenso y observación, respectivamente. Considerando los mismos métodos y el mismo orden, las probabilidades de que una relación fuera de "mediana vida" fueron de $.26, .28$ y .18. Mientras que las probabilidades de que una relación fuera "breve" fueron altas, .58 en nominación, .55 en consenso y .64 en observación.

Propiedades conductuales: inicios e intervalos de interacción

Se aplicó un ANOVA mixto para valorar diferencias a través de los cortes y entre los grupos de relaciones "estables", "mediana vida" y "breves, respecto a los inicios y tiempo de interacción (ver figura 1). 
Comparación de propiedades conductuales en tres grupos de relaciones sociales

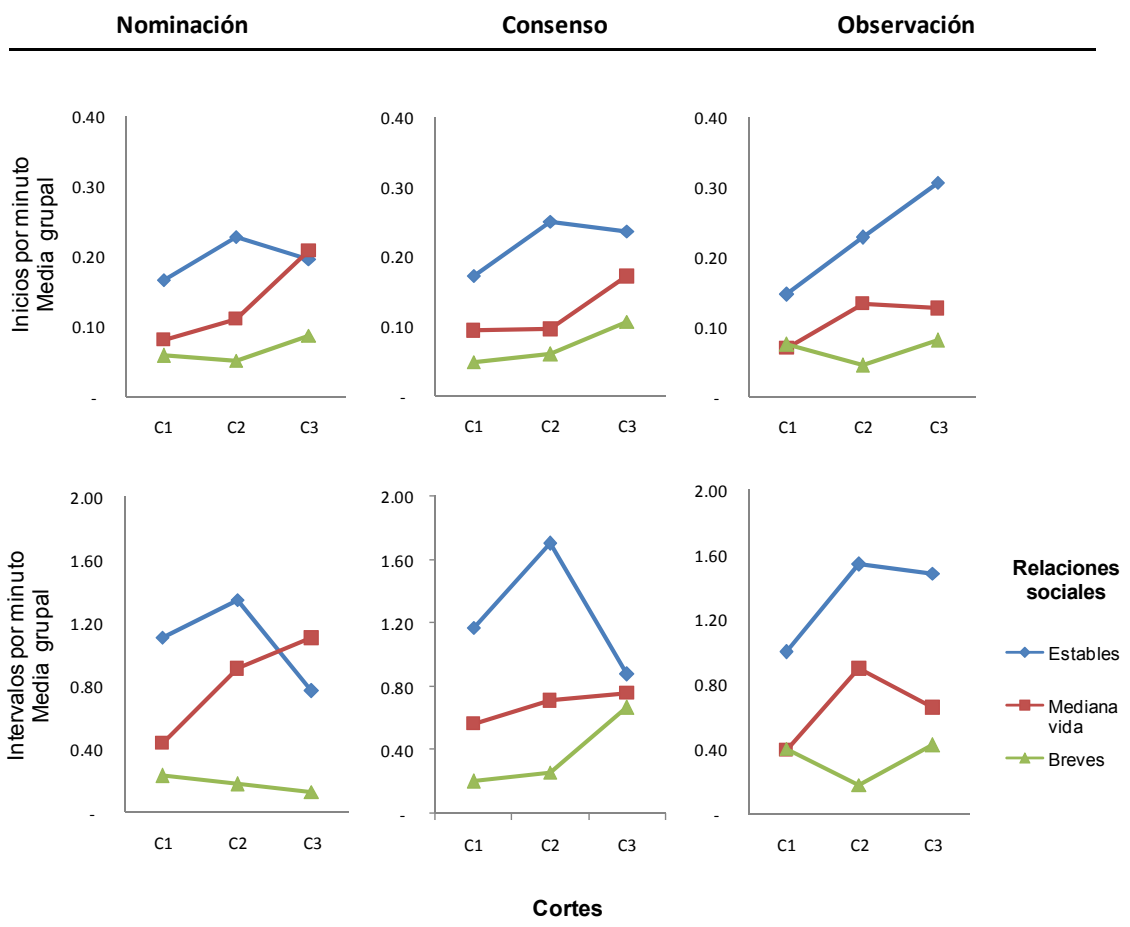

Figura 1. Se comparan dos propiedades conductuales, inicios e intervalos por minuto, de tres grupos de relaciones: estables, mediana vida y breves. $A$ través de tres cortes y en tres métodos que identifican relaciones sociales.

Se encontraron efectos de corte en la proporción de inicios emitidos en las diadas identificadas por nominación $\left(F_{(2.58)}=3.436^{*}, p=.05\right)$, consenso $\left(F_{(2,66)}=3.834, p=.03\right)$ y observación $\left(F_{(2,76)}=6.371^{*}, p=.02\right)$; así como diferencias en la cantidad de inicios entre los grupos de relaciones (estables, mediana vida y breves) en los métodos de nominación $\left(F_{(2,35)}=9.164, p<.01\right)$, consenso $\left(\mathrm{F}_{(2,33)}=11.548, \mathrm{p}<.01\right)$ y observación $\left(\mathrm{F}_{(2,46)}=60.809, \mathrm{p}<.01\right)$. Esta diferencia entre grupos de relaciones también está presente para la proporción de tiempo de interacción para los tres métodos [nominación $\left(F_{(2,35)}=13.279\right.$, $p<.01)$, consenso $\left(F_{(2,33)}=7.365, p<.01\right)$ y observación $\left.\left(F_{(2,46)}=32.591^{*}, p<.01\right)\right]$. Los valores de $F$ marcados con asterisco corresponden al estimador Greenhouse-Geisser por no cumplirse la suposición de normalidad. 
Para valorar específicamente entre qué grupos y cortes se encontraban las diferencias se aplicó un análisis de comparaciones múltiples de Scheffé. Este análisis mostró diferencias entre el grupo de relaciones "estables" y los grupos de "mediana vida" y "breves" en los cortes uno y dos; las relaciones "estables" presentaron mayor frecuencia de inicios e intervalos por minuto, en todos los métodos. En el corte tres las relaciones "estables" exhibieron mayor frecuencia de inicios por minuto sólo en el método de observación. Entre los grupos de "mediana vida" y "breves", sólo se encontraron diferencias respecto a la frecuencia de intervalos por minuto en el corte dos y tres, en los métodos de nominación y observación.

En general, en los tres métodos, se encontró que las díadas de niños que integran las relaciones "estables" hacen más intentos por iniciar interacciones sociales y pasan más tiempo de interacción, en comparación con las díadas de niños que forman las relaciones de "mediana vida" y "breves".

\section{Mecanismos funcionales}

La aplicación de un ANOVA mixto no mostró diferencias significativas entre los grupos de relaciones, en los mecanismos de efectividad y correspondencia. Se observaron altos índices de estos mecanismos en los tres grupos con un índice promedio de .93 para efectividad y .95 para correspondencia, en los tres métodos y cortes de identificación. Por lo tanto, una vez que algún miembro de la díada le habla a su compañero es altamente probable que se involucren en interacción social, independientemente de la duración de la relación.

Con base en un análisis de regresión lineal, se valoró la frecuencia relativa de intervalos en función de la frecuencia relativa de inicios de los tres grupos de relaciones, en los tres métodos de identificación. Con este análisis se valoró la reciprocidad entre lo que cada niño aporta a la relación -sus intentos por iniciar interacción- y lo que recibe -pasar tiempo en interacción-.

El análisis de regresión lineal mostró que las relaciones "estables" tuvieron valores de $\mathrm{R}^{2}$ mayores a los de las relaciones de "mediana vida" (excepto en el método de nominación) y a los de las relaciones "breves". Por lo tanto, en las relaciones "estables" hubo una mayor correspondencia entre lo que cada niño inició y el tiempo de interacción que obtuvo, mientras que en las relaciones "breves" se encontró menor correspondencia entre lo que da y recibe cada integrante de la díada (ver figura 2). 


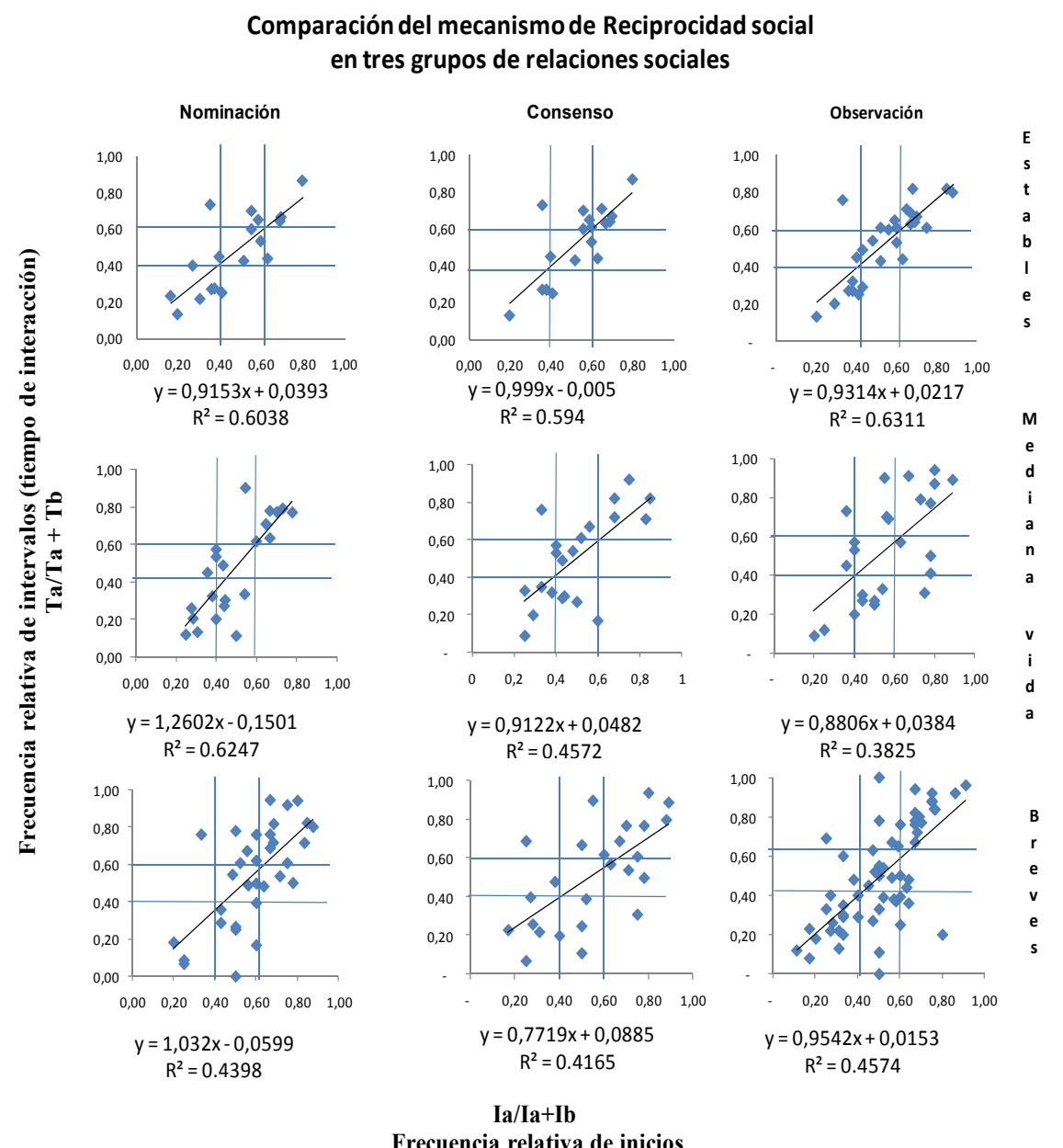

Figura 2. En el eje $X$ aparece la frecuencia relativa de inicios producidos por los integrantes de cada relación y en el eje $Y$ la frecuencia relativa de intervalos generados por tales inicios. Los valores de la $R^{2}$ refieren a la reciprocidad entre lo que cada niño da-esfuerzo por iniciar interacción- y lo que recibe-tiempo de interacción. Las relaciones que aparecen entre los valores .40 y .60 en el eje $X$ son recíprocas en inicios y en el eje $Y$ son recíprocas en intervalos. 
En el grupo de relaciones de "mediana vida" la reciprocidad varió según el método. En el método de nominación la $\mathrm{R}^{2}(.62)$ mostró mayor correspondencia entre iniciativas y consecuencias relativas para cada integrante, aunque el valor de la pendiente 1.26 sugiere que las díadas tendieron a iniciar más y a conseguir, en proporción, menos tiempo de interacción. Para el método de observación se identificó la $\mathrm{R}^{2}$ más baja (.38), y una pendiente de .88 , que sugiere una sub-igualación entre la frecuencia relativa de inicios y la frecuencia de intervalos; por lo tanto, en estas díadas los niños con pocos inicios, alcanzaron más tiempo de interacción.

Otra forma de evaluar la reciprocidad, fue comparar las aportaciones de cada integrante de una relación. Con este análisis se encontró que las díadas podían ser recíprocas en sus inicios (intentos por iniciar interacción) y/o en el tiempo de interacción asignado al compañero como consecuencia de esos inicios. Las relaciones recíprocas en inicios aparecieron entre los valores de .40 y .60 del eje $X$, las relaciones recíprocas en intervalos se encontraron entre los valores de .40 y .60 en el eje $\mathrm{Y}$, y las relaciones ubicadas entre estos valores en ambos ejes, cuadrante central, fueron recíprocas tanto en inicios como en intervalos.

La mayor proporción de relaciones recíprocas en inicios, se presentó en el grupo de relaciones de "mediana vida". La proporción de díadas recíprocas en intervalos, en general fue baja para todos los grupos de relaciones. La proporción de díadas recíprocas tanto en inicios como en intervalos, fue mayor en el grupo de relaciones "estables" en todos los métodos, lo cual sugiere que en éstas, ambos integrantes iniciaron interacciones en proporciones similares y el tiempo de interacción que generó cada uno también fue similar.

\section{Desarrollo de las relaciones sociales}

La probabilidad de que una relación fuera "estable" se analizó con base en el número de métodos que la identificaron. La probabilidad de que una relación fuera "estable" en nominación fue de .60 cuando ésta era identificada por los tres métodos en el corte uno, mientras que en las díadas que sólo fueron identificadas por nominación o por nominación junto con otro método, la probabilidad de que dos niños volvieran a nominarse como amigos fue de cero. Las mismas probabilidades se encontraron en el método de consenso. En el método de observación, la probabilidad de que una relación fuera "estable" en observación fue de .50 sí fue identificada por los tres métodos, de .67 sí fue identificada por observación y consenso, y de .11 sí fue identificada sólo por observación. En la figura 3 aparecen tres diagramas de conjuntos que representan la convergencia entre los tres métodos de identificación. 


\section{Covergencia entre métodos de identificación}

Diversidad de relaciones sociales entre niños

\section{Corte 1}

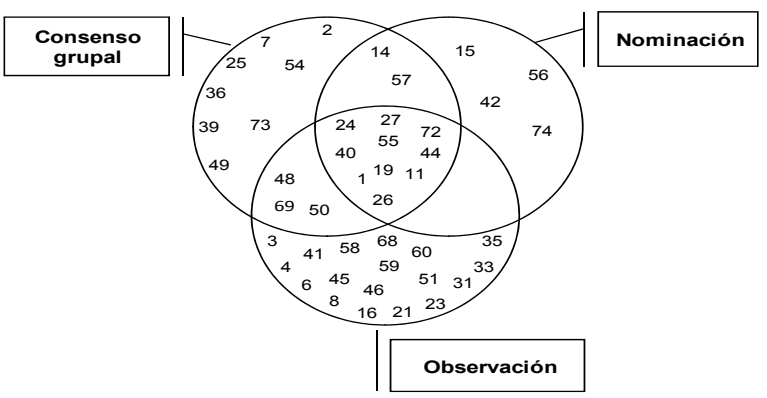

Corte 2

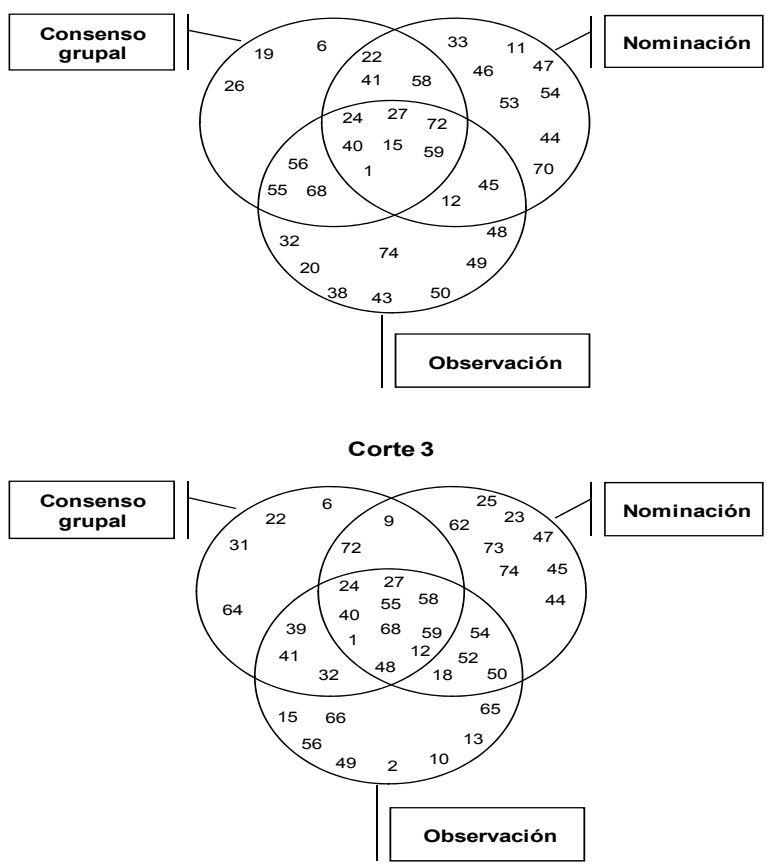

Figura 3. Los círculos representan cada uno de los métodos para identificar relaciones sociales y los números representan a cada una de las relaciones sociales. Las intersecciones contienen a las díadas identificadas por dos o tres métodos en cada uno de los cortes. 
Con el análisis de convergencia se reconocieron tres agrupaciones de relaciones que fueron identificadas por los tres métodos en el corte uno, pero que mostraron diferente desarrollo. La primera agrupación la conformaron las díadas 1, 24, 27 y 40, las cuales fueron relaciones "estables" en todos los métodos, es decir, en los tres cortes los niños se nominaron mutuamente como amigos, por consenso eran "niños que se juntan mucho" y fueron observados interactuando. La segunda agrupación la integraron las díadas 44, 55 y 72 , que resultaron ser estables en uno o dos métodos. La tercera agrupación se compuso por las díadas 11,19 y 26 , éstas se manifestaron como relaciones de "mediana vida" con un decremento importante en sus intervalos de interacción. Lo anterior implica que el $70 \%$ de las relaciones identificadas por los tres métodos fue "estable" y el $30 \%$ fue de "mediana vida", al menos en una de las mediciones.

Finalmente, se identificó un número muy reducido de díadas mixtas (niña-niño), las cuales se encontraron dentro del grupo de relaciones "breves". Todas las relaciones "estables" estuvieron constituidas por niños del mismo género.

\section{DISCUSIÓN}

En este estudio se encontraron proporciones de relaciones estables -identificadas por nominación mutua- inferiores a las encontradas en otros trabajos (Rubin, Wojslawowicz, Rose-Krasnor, Booth-LaForce \& Burgess, 2006). Tales diferencias pueden ser atribuidas a los criterios para seleccionar a las relaciones. La mayoría de los estudios limitan las nominaciones sólo a tres o dos "mejores amigos" o bien, a compañeros del mismo género. En cambio, en la presente investigación no se limitó el número de compañeros que los niños podían nominar ni el género de los mismos. Dichos criterios influyen en la cantidad total de díadas identificadas sobre la cual se estima la proporción de díadas que son estables; es posible que al limitar el número de relaciones que los niños deben reportar se sobre estime la estabilidad de las mismas.

En cuanto a la estabilidad de las relaciones identificadas mediante los mapas socio-cognitivos compuestos, sólo se ha evaluado la estabilidad de la configuración del grupo, más no la estabilidad de las díadas (Cairns et al, 1995). Los hallazgos sobre la estabilidad de relaciones sociales identificadas por observación son consistentes y similares en porcentaje con los encontrados por Barbu (2003).

En general, al igual que en otros estudios, se encontró que los niños se involucran al mismo tiempo en varias relaciones, construyen nuevas y algunas las abandonan (Barbu, 2003; Wojslawowicz, et al., 2006). Independientemente del método de identificación y de que el contexto escolar mantenga 
la configuración de los grupos, la estabilidad de las relaciones en periodos mayores a seis meses es un fenómeno que ocurre poco entre niños de 10 años de edad.

Los resultados apoyan que las relaciones con altas frecuencias de tiempo de interacción y las díadas constituidas por niños del mismo género son más estables, lo que es consistente con otras investigaciones (Barbu, 2003). Las propiedades de inicio y la reciprocidad, se asocian a la estabilidad de las relaciones. Así, aquellas relaciones con mayor frecuencia de interacción social e inicios, tendrán mayor probabilidad de ser "estables" en sus nominaciones de amistad, en altas frecuencias de interacción y en que sus compañeros los reconozcan como "niños que se juntan mucho". Las relaciones "estables" tienden a ser recíprocas, además de que obtienen consecuencias de interacción proporcionales a sus actos de inicio.

En relación a los mecanismos de efectividad y correspondencia, estos hallazgos contribuyen a reafirmar el papel que tienen dentro de las experiencias sociales entre niños. Se requiere de alguien que inicie el contacto social y de alguien que conteste para que las interacciones ocurran y se construyan relaciones. Por lo tanto, su función parece estar asociada al establecimiento de interacciones o vínculos, pero no necesariamente al mantenimiento de las relaciones sociales (Santoyo, Colmenares \& Losada, 2007).

En este trabajo se considera que los llamados mecanismos funcionales son referentes conductuales, que muestran cómo se integran las competencias de ambos integrantes. Son indicadores de la función que tiene el acto social de un niño sobre la conducta de otro, ya que permiten observar si el acto de inicio de un niño lo llevó a acceder a un recurso social -interactuar con un compañero- y más aún, en qué proporción consiguió este recurso. Conjuntamente, valoran sí el niño es capaz de ofrecer recursos sociales a otros y en qué proporción respecto a su compañero.

Por lo tanto, es importante considerar cuando se diseñan programas de capacitación en habilidades sociales, que existen diferentes niveles de complejidad social, que si bien están relacionados, implican procesos particulares. Un programa puede estar diseñado para enseñar a un niño a iniciar e involucrase en interacciones sociales, pero no implica que con ello se estén proporcionando los recursos para mantener una relación social (Asher, et al., 1996). Así, se requiere de mecanismos adicionales, como el de la reciprocidad, para que una relación social entre niños sea estable, al menos a lo largo de un año.

Al analizar la convergencia y divergencia entre métodos de identificación, se apreciaron dos importantes hallazgos. Primero, las relaciones sociales identificadas por los tres métodos tendrán una probabilidad muy alta de ser estables por un año o al menos seis meses. Segundo, el hecho de que las diferentes mediciones no coincidan, significa que cada uno de ellas mide pro- 
piedades distintas, las cuales no necesariamente cambian o se mantienen al mismo tiempo.

El empleo de tres procedimientos también muestra que se debe ser cauto cuando se interpreta la información obtenida de cada método. Es un riesgo considerar que todas las díadas que se identifican por observación son relaciones de amigos (Smith \& Connolly, 1980), decir que todos los amigos pasan mucho tiempo de interacción, o creer que los informantes externos siempre son sensibles a los cambios en las relaciones de otros; situación que se observó en una de las relaciones, cuando dos niñas dejaron de involucrarse en interacciones sociales aunque sus compañeros seguian reportando que se juntaban mucho.

Una perspectiva dinámica de las relaciones sociales permite ampliar el espectro de análisis y considerar las transformaciones que vive una díada en el tiempo. Por ejemplo, es probable que la denominación de amistad unilateral, implique relaciones en proceso de consolidación, es decir, que recién inician y posteriormente ambos niños llegarán a llamarse amigos. Pero también es posible que estas relaciones unilaterales impliquen relaciones que están por disolverse, por ello sólo uno de los integrantes menciona al otro como su amigo.

\section{REFERENCIAS}

Asher, R.S., Parker, G.J. \& Walker, L.D (1996). Distinguishing friendship from acceptance: Implications for Intervention and assessment. En W.M. Bukowski, A.F. Newcomb \& W.W. Hartup (Eds.), The Company they keep: Friendship in childhood and adolescence (pp.366-405). New York: University Press.

Barbu, S. (2003). Stability and flexibility in preschoolers' social networks: A dynamic analysis of socially directed behavior allocation. Journal of comparative psychology, 117(4), 429-439.

Berndt, T. J. \& Hoyle, S. G. (1985). Stability and change in childhood and adolescent friendships. Developmental Psychology, 21(6), 1007-1015.

Bukowski, W. M. \& Newcomb, A. F. (1984). Stability and determinants of sociometric status and friendship choice: A longitudinal perspective. Developmental Psychology, 20(5), 941-952.

Cairns, R. B., Leung, M., Buchanan, L. \& Cairns, B. D. (1995). Friendships and social networks in childhood and adolescence: fluidity, reliability, and interrelations. Child Development, 66, 1330-1345.

Espinosa, A.M.C, Blanco-Villaseñor, A. \& Santoyo, V. C. (2006). El estudio del comportamiento en escenarios naturales: El sistema de observación de las interacciones sociales. En C. Santoyo y C. Espinosa (Eds.) Desarrollo e interacción social: Teoría y métodos de investigación en contexto. México: UNAM/CONACyT. 
Gest, S. D., Farmer, T. W., Cairns, B.D \& Xie, H. (2003). Identifying children's peer social networks in school classrooms: Links between peer reports and observed interactions. Social Development, 12(4), 513-529.

Gottman, J. M. (1979). The structure of interaction. En J.M. Gottman (Ed), Marital Interaction: Experimental investigations (pp. 45-76). New York: Academic Press.

Hinde, R., A. (1995). A suggested structure for a science of relationships. Personal relationships, 2, 1-15.

Kohler, F.; Phillips, S.; Hoyson, M.; Davis, L.; Donina, W.; \& Rapp, N. (1995). Using a group-oriented contingency to increase social Interactions between children with autism and their peers. Behavior Modification, 19, 10-32.

Rubin, K. H., Bukowski, W. \& Parker, J.G. (1998). Peer interactions, relationships, and groups. En W. Damon \& N. Eisenberg (Eds.) Handbook of child psychology Vol. 3 (pp. 619-700). New York: J. Wiley.

Rubin, K. H., Wojslawowicz, J.C., Rose-Krasnor, L., Booth-LaForce, C. \& Burgess, K.B. (2006). The best friendships of shy/withdrawn children: Prevalence, stability and relationship quality. Journal of abnormal child psychology, 34(2), 143-157.

Santoyo, V. C. (2006). La ecología social de la cotidianeidad en la escuela: Redes sociales y mecanismos funcionales. En C. Santoyo y C. Espinosa (Eds.) Desarrollo e interacción social: Teoría y métodos de investigación en contexto (pp.113-150). México: UNAM/CONACyT.

Santoyo, V. C., Colmenares, V. L. \& Figueroa, B. N. (2007). Estabilidad y cambio de patrones coercitivos: Un enfoque de síntesis. En C. Santoyo (Ed.) Estabilidad y cambio de patrones de comportamiento en escenarios naturales: un estudio Iongitudinal en Coyoacán (pp. 113-148). México: UNAM/CONACyT.

Santoyo, V. C., Colmenares, V. L. \& Losada, L. J. L. (2007). Redes sociales: Modalidades, funcionalidad y continuidad en una disciplina de síntesis. En C. Santoyo (Ed.) Estabilidad y cambio de patrones de comportamiento en escenarios naturales: un estudio longitudinal en Coyoacán (pp. 81-112). México: UNAM/CONACyT.

Smith, P. K. y Connolly, K. J. (1980). The ecology of pre-school behavior. Cambridge: University press.

Wojslawowicz, J.C., Rubin, K. H., Burgess, K.B., Booth-LaForce, C. \& Rose-Krasnor, L. (2006). Behavioral characteristic associated with stable and fluid best friendship patterns in middle childhood. Merrill-Palmer Quarterly, 52(4), 671-693. 